\title{
TIRANÍA DEL PAPER. IMPOSICIÓN INSTITUCIONAL DE UN TIPO DISCURSIVO
}

\author{
José Santos Herceg \\ IDEA / USACH \\ santosherceg@gmail.com
}

RESUMEN / ABSTRACT

Este trabajo busca analizar la irrupción de paper como un modo de escritura que en el último tiempo está siendo impuesto en el ámbito de las Humanidades con el apoyo de una serie de instituciones que lo ha hecho posible. El objetivo de este escrito será, por un lado, estudiar sus características principales a partir de la información contenida en los manuales e instructivos para escribir papers y, por otro lado, poner en evidencia sus implicancias para el trabajo intelectual en general y el latinoamericano en particular.

PALABRAS ClaVE: paper, política de publicación, institución, prohibición.

This paper analyzes the emergence of the paper as a kind of writing in the area of the Humanities that, in recent times, and with institutional support, has been imposed as the nearly unique and acceptable kind of academic discourse. The aim of this paper is, first, to study the main characteristics of this kind of writing, based on information in the manuals and instructions for paper writing and, secondly, to signal its implications for the intellectual work in general and for the work in Latin America in particular.

Key words: Paper, publishing policy, institution, prohibition. 


\section{PUESTA EN ESCENA}

La mera constatación del cúmulo de discursos, la enorme cantidad de textos que se almacenan en bibliotecas, librerías, computadores, etc.; así como el continuo y sorprendente caudal de escritos que se producen anual, mensual, semanal y diariamente en los diferentes ámbitos de las Humanidades, será mi punto de partida. Si miramos paradigmáticamente los casos de los estudios literarios y la investigación filosófica, se pone en evidencia este punto: cientos de libros, revistas, conferencias, ponencias, clases. Poniendo como ejemplo el caso de Chile, un país con una "recatada" vida filosófica, que cuenta tan solo 17 centros universitarios donde se cultiva la disciplina ${ }^{1}$ y algo más de 200 académicos activos contratados en dichos centros, tiene, no obstante, más de una treintena de revistas especializadas en Filosofía que se publican regularmente ${ }^{2}$. No se necesita mucha imaginación para proyectar lo que ocurre en un país con una "activa" vida filosófica. Algo análogo podría, sin duda, corroborarse al mirar la situación en otras áreas de la Humanidades. En el caso de los estudios literarios, para traer a colación otro ejemplo paradigmático, tenemos en Chile aproximadamente 15 lugares en que se enseña Literatura como grado académico ${ }^{3}$ y se cultiva la disciplina, y existen, no obstante, más

\footnotetext{
Universidad de Santiago de Chile, Pontificia Universidad Católica de Chile, Pontificia Universidad Católica de Valparaíso, Universidad de Chile, Universidad Metropolitana de Ciencias de la Educación, Universidad Católica del Maule, Universidad Católica del Norte, Universidad de Concepción, Universidad de La Serena, Universidad de Playa Ancha, Universidad de los Andes, Universidad Alberto Hurtado, Universidad Cardenal Silva Henríquez, Universidad Católica de la Santísima Concepción, Universidad Gabriela Mistral y Universidad Andrés Bello.

Archivos, Revista de Filosofia; Filosofía, Educación y Cultura; Veritas, Revista de Filosofia y Teología; Intus-Legere, Revista de Filosofia, Historia y Letras; Seminarios de Filosofia; Nombrada; Anuario de Pregrado; Philosophica; Logos, Revista de Lingüística, Filosofia y Literatura; Límite, Revista de Filosofia y Psicología; Hermenéutica Intercultural; Anales de Filosofía Jurídica y Social, Cuadernos de Filosofia; Diadokhé; Perspectivas Éticas; Teoría del Arte; Paralaje; Cuaderno del Seminario; Margen, Revista de Filosofia y Letras; Observaciones Filosóficas y La Cañada, Revista del Pensamiento Filosófico Chileno.

Las universidades en que se ofrecen licenciatura, magíster y doctorado son: Pontificia Universidad Católica de Chile, Universidad de Chile, Universidad Diego Portales, Universidad Alberto Hurtado, Universidad Finis Terrae, Universidad de los Andes, Universidad de las Américas, Universidad Andrés Bello, Universidad del Desarrollo, Universidad Adolfo Ibáñez, Universidad de Santiago de Chile, Universidad Católica de Valparaíso, Universidad ARCIS. Universidad de Playa Ancha y Universidad de Concepción.
} 
de una treintena de revistas en las que se publican regular y periódicamente estudios literarios ${ }^{4}$.

La cantidad, sin embargo, no es solo el problema, sino que también la variedad y la heterogeneidad en lo relativo al formato. Dicho de otra manera, tomando nuevamente a la Filosofía como ejemplo, es evidente que ella se escribe y se ha escrito de múltiples formas. Como han constatado autoras como Christiane Schildknecht (21) y Alcira Bonilla (42) o, antes que ellas, Julián Marías (334-5); entre los múltiples rostros de la forma literaria de la Filosofía podrían mencionarse el diálogo, el aforismo, la carta (auténtica o ficticia), el ensayo, la plegaria, la meditación, el fragmento, el manual, los poemas, las diatribas, las biografías y las autobiografías, los ensayos, las meditaciones, los relatos utópicos, las exhortaciones, las summa y los tratados, los artículos y los papers, entre otros. En el ámbito de los estudios de la Historia se da también esta pluralidad de modos de escritura. De hecho, en este ámbito autores como Hayden White han estudiado la Historia directamente como discurso y otros han llegado a sugerir que la tarea de distinguir entre discurso histórico y discurso ficcional es tan ardua como imposible (Cf.: Ricouer). En Historia se pueden encontrar tratados, ensayos, artículos, (auto)biografías, crónicas, pero también, han sostenido algunos, hay Historia en las llamadas novelas históricas (Cf.: Pizarro Cortés). Jaques Le Goff ha llegado incluso a hablar de un "ensanchamiento de la noción de Historia" que implica incorporar como historiografía el cine, la televisión, la radio, la prensa, etc. (50).

Las Humanidades, las ciencias humanas o del espíritu ${ }^{5}$ están constituidas, entonces, por un conjunto de discursos que, tanto por su cantidad como

$4 \quad$ Revista Chilena de Literatura, Acta Literaria, Atenea, Taller de Letras, Estudios Filológicos, Literatura y Lingüistica, Anales de la Literatura Chilena, Alpha, Aiesthesis, Revista Laboratorio, Analecta, Revista de Humanidades UNAB, Revista de Estudios Avanzados, Revista Universum, Revista Grifo, Crítica.cl, Revista literario contrafuerte, Letras de Chile, Acta literaria, Letras S5, Rocinante, Revista literaria de la Sociedad de Escritores de Chile, Documentos lingüisticos y literarios, Revista Signos, Mapocho, Dossier, Revista Chilena de Humanidades, Actas de Lengua y Literatura Mapuche.

Utilizaré indistintamente los términos Humanidades, ciencias humanas y ciencias del espíritu. A sabiendas de que es posible establecer algunas distinciones entre ellas, sin embargo, a lo que se aludirá con estas denominaciones es a aquellas disciplinas que no son parte de las "Ciencias Naturales" ni pueden inscribirse bajo el rótulo de "Ciencias Sociales". Entre ellas habría que considerar a la Filosofía, los Estudios Literarios, la Historia, los Estudios Culturales, etc. 
por la diversidad de sus formatos, se ha vuelto prácticamente inabarcable. Esta constatación habría que empatarla con otra igualmente ostensible: la existencia de una cierta organización de este mundo discursivo. Organizar digo - para comenzar con cautela - aludiendo al modo en que se administra esta irrupción descontrolada y descomunal de discursos. La distinción misma entre diferentes géneros o modos de escritura es ya una suerte de organización, de administración de los discursos que los agrupa de acuerdo con sus formas narrativas o según los tipos de textos. La jerarquización de los discursos es también otra forma de organización del panorama discursivo. Es así como hoy por hoy, por ejemplo, es casi evidente que a un poema se le niegue a priori su carácter de texto filosófico o histórico, a un diálogo o a una autobiografía al menos se le ponga en duda su carácter de estudio literario, a un ensayo se le podría aceptar a regañadientes su lugar en el ámbito de la Filosofía, pero no así en el de la Literatura y la Historia, y a un libro -un tratado-, un artículo o un paper se les considera aceptados en general en la Humanidades, casi sin discusión. El gesto de Francisco Romero, al establecer que ciertos tipos de textos son los "normales" para la filosofía en América Latina podría entenderse, así, como un gesto administrativo extensible a todos los otros ámbitos de las Ciencias Humanas.

La organización de los discursos tiene, de este modo, en un primer y evidente sentido, un carácter jerarquizante y marginante: ciertos discursos son aceptados; otros no lo son. La noción de "archivo" acuñada por Foucault pude ser útil para comprender este punto. Como explica el francés, Archivo sería la " $(. .$.$) ley de lo que puede ser dicho, el sistema que rige la aparición$ de los enunciados" (Arqueología 219), el "sistema general de la formación y de la transformación de los enunciados" (Arqueología 221). El Archivo no es algo que se pueda describir cabalmente, pero lo que sí es claro es que pertenece a una sociedad, a una cultura, a una época determinada. Lo que pueda ser enunciado en el campo de las ciencias humanas depende, según él, del Archivo correspondiente, pues este establece las normas que determinan esa "práctica discursiva". Archivo es aquel "(...) conjunto de reglas anónimas, históricas, siempre determinadas en el tiempo y el espacio que han definido en una época dada, y para un área social, económica, geográfica o lingüística dada, las condiciones de ejercicio de la función enunciativa"(Arqueología 198).

En una primera mirada me ha parecido que dicha "organización" de los discursos, que la existencia de un "archivo" tenía sentido en virtud de la necesidad de poner orden en el caudal discursivo, con el objeto de hacer más efectiva, eficiente-incluso simplemente posible-la labor de los investigadores, 
así como también la de las instituciones vinculadas con el saber filosófico y la asignación de recursos (Cf.: Santos Herceg, Espejismos). A lo que me refiero es, por ejemplo, a aquella necesidad de poder discriminar entre discursos de acuerdo con su calidad, para así abocarse solo al trabajo sobre lo que realmente aporta al conocimiento o a la necesidad de evaluar el trabajo o la trayectoria de un determinado investigador para otorgar becas, financiamiento o puestos de trabajo. En otro contexto, de lo que estoy hablando es de aquello que hoy se conoce con el nombre de "cienciometría" (Cf. Spinak).

En una segunda mirada me va pareciendo más acertado pensar que, en la medida en que se ha instalado una determinada idea acerca de lo que es el "saber" en el ámbito de las Humanidades, dicha concepción va respaldada, fortificada, si se quiere, por una cierta institucionalidad que exige una organización desde donde se impone un determinada administración de los discursos. Hay envuelto aquí un elemento central que, por sus consecuencias, interesa que sea destacado. A lo que me refiero es al papel del temor, del miedo presente en este esquema de organización y administración, detrás del cual late un espíritu reaccionario y controlador. La sospecha aquí es que la finalidad de esta organización es aligerar de su peligrosidad al discurso que circula sin restricciones, controlando, ordenando, lo que Foucault llama "los juegos del pensamiento y de la lengua" ". El filósofo francés habla de que en el fondo late una "Logofobia" - oculta subrepticiamente tras una aparente "Logofilia"7. El paper, tema que se abordará ahora, me parece un caso paradigmático de esta "logofobia", un ejemplo actual y contundente de una forma de controlar la peligrosidad del discurso de las Humanidades en general, de dominar su proliferación, de organizar su incontrolabilidad mediante prohibiciones, barreras, límites y reglas.

\footnotetext{
"Todo pasa como si prohibiciones, barreras, límites, se dispusieran de manera que se domine, al menos en parte, la gran proliferación del discurso, de manera que su riqueza se aligere de la parte más peligrosa y que su desorden se organice según figuras que esquivan lo más incontrolable; todo pasa como si se hubiese querido borrar hasta las marcas de su irrupción en los juegos del pensamiento y de la lengua" (Foucault, Orden 50).

“(...) una especie de sordo temor contra esos acontecimientos, contra esa masa de cosas dichas, contra la aparición de todos esos enunciados, contra todo lo que pueda haber allí de violento, de discontinuo, de batallador, y también de desorden y de peligro, contra ese gran murmullo incesante y de desordenado de discurso" (Foucault, Orden 51).
} 


\section{PERSONAJE PRINCIPAL}

El paper, en tanto que uno más de los géneros posibles de escritura, se ha ido instalando de un tiempo a esta parte como el modo privilegiado de escritura en el ámbito de las Ciencias Humanas. Dicha instalación no se debe, sin embargo, a que este formato, este género literario sea "señalado", para utilizar un término heideggeriano y que por ello, últimamente se haya ido imponiendo de forma natural como el formato más idóneo para las Humanidades y, valga decir, para todos los ámbito del saber. La realidad es que las instituciones han llevado a cabo una sistemática campaña con vistas a imponer este modo de escritura como el prioritario, el mejor evaluado, el más deseable, e incluso, en algunos casos, como el único aceptable. En el caso chileno, que es por supuesto el más cercano para el autor de este estudio, esta "campaña" en vistas de priorizar el paper es evidente. Como es bien sabido por todos quienes se dedican a la investigación en mi país, el Fondo Nacional de Desarrollo de las Ciencias y la Tecnología (FONDECYT) es la institución pública que financia proyectos de investigación a la que la gran mayoría - por no decir todos- los investigadores nacionales postulan. Lo interesante de observar aquí es que, al menos hasta el 2011, en la selección de los proyectos, la distribución de puntajes asigna $40 \%$ al curriculum del investigador, y de ese $40 \%$, el $60 \%$ es por "productividad", es decir, textualidad publicada. Lo relevante para efectos de lo que vengo diciendo es que aquello a lo que más puntaje se le asigna es a los papers -en revista ISI, 10 puntos, en revistas Scielo, 7 puntos- incluso más que a la publicación de un libro ${ }^{8}$.

Lo descrito respecto de FONDECYT no es una excepción: se trata solo de un girón de todo un sistema que ha ido instalando sistemáticamente en Chile la producción de papers como algo prioritario. Es así como en la asignación de becas para estudios de posgrado, la Comisión Nacional de Ciencia y Tecnología (CONICYT) utiliza estos mismos criterios, y también las universidades, desde hace un tiempo, han decidido implementarlos. De hecho, para determinar quiénes son los investigadores destacados, a quiénes se los premia incluso con incentivos económicos, se observa prioritariamente la producción de papers y el lugar en el que han sido publicados. Estos criterios

Solo en casos excepcionales, por acuerdo entre los miembros del Grupo de Estudio, se puede llegar a asignar hasta 12 puntos por un libro publicado en una editorial de circulación internacional, que posea comité editorial. 
se utilizan además, por el otro extremo, para justificar la separación de algún académico del plantel dada su baja productividad o para contratar a un nuevo elemento. Pero no solo eso, las universidades utilizan estos criterios también para evaluar su propio desempeño en investigación: ellas se miden a sí mismas en virtud de la producción de papers. Esto, por supuesto, no es casual, pues ningún otro tipo de discurso, solamente la producción de papers, tiene una repercusión -y sustantiva- en la asignación del Aporte Fiscal Directo 9.

No hay puntaje en FONDECYT cuando se postula a un proyecto, ni en CONICYT cuando se solicita una beca si se presentan ensayos, diálogos o biografías como producción. Ni siquiera se considera un espacio en los formularios para ingresar como productos de la reflexión los aforismos que se hubieran acuñado, ni los fragmentos o poemas escritos. Los tratados, los libros, los textos de largo y trabajoso aliento tienen una valoración menor, y eso, cuando la tienen. Dicha valoración, además, no depende de su contenido, sino de la editorial en que son publicados. De acuerdo con los parámetros que se están imponiendo hoy para la evaluación de la producción, la publicación de la Crítica de la Razón Pura no habría traído grandes beneficios ni a Kant ni a la Universidad de Königsberg, ni la de Ser y Tiempo habría ayudado a una mejor valoración del trabajo de Heidegger. De igual manera, en el ámbito local la Reflexión Cotidiana, el maravilloso escrito de Humberto Giannini, no aporta gran cosa para asegurar que este filósofo obtenga financiamiento para investigar. De hecho, en una oportunidad Fondecyt le rechazo una postulación. La priorización de los papers ha conducido, en la práctica, a una situación francamente ridícula en que investigadores noveles o no tan noveles, pero con gran producción en términos de papers, obtienen apoyo para investigar sin problemas, mientras que autores de referencia y consagrados no logran figurar en la nómina de los ganadores de financiamientos.

Pero, ¿qué es un paper?, ¿qué es esta textualidad que se nos está imponiendo como la prioritaria, como la más adecuada, como la única aceptable? La pregunta puede parecer sorprendente, pero, como suele ocurrir, se trata de aquellas cosas que se supone todos sabemos. De hecho, lo sabemos, pues escribimos papers, pero ante esta pregunta no sabemos bien qué decir. Se

El Decreto Supremo de Educación No 128, de 1991, establece que el monto del Aporte Fiscal Directo que otorga el Estado a las instituciones de Educación Superior depende en un $33 \%$ de la cantidad de papers publicados en revistas ISI y el Decreto $\mathrm{N}^{\circ} 116$ de 2002 agrega que este dependerá, además, en un $11 \%$ de lo que aparezca en revistas SCielo, lo que da como resultado que el monto se juega en un $44 \%$ en la publicación de papers. 
podría hablar aquí de una especie de "doble saber" en un sentido análogo al usado por Wittgenstein: un saber que se tiene y que se refiere al hacer, y un saber que no se tiene referido al explicar. Sabemos escribir un paper, lo hacemos con mayor o menor facilidad, pero lo hacemos. Si nos preguntan, sin embargo, qué es lo que caracteriza a un paper, no tenemos tan claro qué responder. La respuesta, por lo tanto, se vuelve complicada a primera vista. Partamos, entonces, por lo más evidente: para nadie es un secreto, pues es una cuestión bastante conocida, que el paper es un género de escritura que se desarrolla en el contexto de las Ciencias Naturales y responde a un determinada idea del saber científico que encuentra en este tipo de discurso el medio más idóneo para la comunicación. La instalación del paper en el ámbito de las Humanidades, por lo tanto, obedece a un "traslado", para decirlo con delicadeza, o a una "invasión" si se quiere ser más insidioso. Sin detenerme en este punto, que ya ha sido esbozado antes por otros, quisiera simplemente comenzar destacándolo con el objeto de poner en evidencia que la irrupción de paper en el ámbito de las Humanidades es más bien tardía, de hecho, me atrevería a llamarla, reciente.

Antes de la aparición del paper, en las ciencias del espíritu ya existía un tipo de discurso que comparte con él algunas características, lo que ha llevado a confusiones que sería importante aclarar. "Artículo" en español, "Aufsatz" en alemán, "Article" en francés, son términos que no pueden considerarse, sin más, como sinónimos de paper. Quisiera sostener aquí la tesis de que el paper se ha incorporado al contexto de los géneros literarios de las Humanidades como una especie, como un tipo de artículo, ese que algunos han llamado también "artículo académico" o "artículo científico". Las características del paper, por lo tanto, no deben extenderse a todo tipo de artículo, pues las alternativas para cultivar este género son más amplias. Cuando se sostiene, entonces, que el paper se ha ido instalando como el modo privilegiado de escritura, y ello con el apoyo de toda una institucionalidad, debemos tener claro que esto no se aplica a todo tipo de artículo, sino solo al paper: aquel texto breve cuyo origen se encuentra en las Ciencias Naturales y que, instalado en el ámbito de las Humanidades, adquiere características bien nítidas que pueden ser descritas, sin mayor dificultad con ayuda de la gran cantidad de "manuales", de "instructivos", de "consejos" acerca de cómo escribir un paper que inundan Internet ${ }^{10}$. 
Una de las características más interesantes de resaltar respecto del paper es que se trata de un escrito fundamentalmente efímero y su contenido, por lo tanto, también lo es. Un paper que contenga nuevos contenidos, nuevos productos de investigaciones más avanzadas, más modernas, más cercanas a la verdad, habría de dejar atrás los conocimientos antiguos, superándolos. Así, un paper de hace 20 años está desactualizado, obsoleto, ha sido superado en estos años por otros muchos con sus nuevos enfoques y verdades, con datos actuales e información fresca. La viabilidad de una investigación propuesta se evalúa, entre otras cosas, por la actualidad de los textos consultados, por lo moderno de su bibliografía.

Esto, que tal vez podría corroborarse en Ciencias Naturales, en el caso de las Humanidades es, sin duda, un tema de discusión. La aparición del paper implica la instalación de lo efímero en el ámbito de las Humanidades, de la idea de superación de lo antiguo por lo moderno -siendo lo antiguo algo de solo unos años o incluso meses de antigüedad. Esto contrasta, sin duda, con la idea de "tradición" y con la existencia de los textos considerados "clásicos". La tradición reconoce discursos con eminente valor, con claros aportes y reconocida actualidad -clásicos- que fueron escritos hace cientos e incluso miles de años y que, en ningún caso, están obsoletos. Los ejemplos casi no son necesarios de traer a colación: en el caso de la Filosofía bastaría con aludir, por ejemplo, al hecho evidente de que el Discurso del Método no ha dejado atrás a los Diálogos platónicos ni a la Metafísica de Aristóteles, del mismo modo como las Investigaciones lógicas de Husserl no hacen olvidar los aportes y el valor de la Summa Teológica de Tomás de Aquino. En el contexto latinoamericano, autores como Alberdi, Sarmiento, de Hostos, Fuentes, Echeverría, Bilbao, Bello, Ardao, Salazar Bondy, Zea, Francovic, Henríquez Ureña, Millas, Giannini, Schwarztman, entre tantos otros, han producido obras imperecederas, sin duda clásicas.

La "humildad" o "modestia" son otros rasgos característicos del paper. Quizás sea por lo breve de su extensión, en la que no alcanza a desarrollarse latamente una tesis y, mucho menos, múltiples hipótesis, que este tipo de textos se propone simplemente trabajar una idea aislada, un aspecto de un problema, un escorzo, un matiz. Quienes elaboran manuales dirigidos a la formación de estudiantes y que pretenden enseñar a redactar un paper insisten en que, "A good paper is modest and makes a small point (...)" (Pryor). Esta modestia se refleja también en los límites que reconoce en cuanto a sus pretensiones; dicho coloquialmente, nadie puede pretender, ni pretende, en el marco de un paper, cambiar la historia del pensamiento, ni siquiera desarrollar una teoría propia. 
Esta humildad de pretensiones, claramente es una actitud nueva en el ámbito de las Humanidades, pues, como se decía, el paper es un género de aparición reciente. Lo que prima entre los autores desde la Antigüedad hasta bien entrado el siglo XX, son principalmente los textos de largo aliento, como los tratados, los libros de diferentes tipos y diversos estilos, donde un autor espera dar cuenta acabada, cabal e incluso total de un tema. Kant -nada de humilde en sus pretensiones-dice, por ejemplo, expresamente en la Crítica de la Razón Pura que cree haber resuelto "todos" los problemas de la Metafísica $\mathrm{o}$, al menos, haber puesto las bases para hacerlo ${ }^{11}$.

El supuesto de la construcción de saber a partir de los papers es que se suman, que se va construyendo en la medida en que los nuevos trabajos se basan y superan a los más antiguos. Esto tal vez sucede efectivamente en las Ciencias Naturales, pero en el ámbito de las del Espíritu, la construcción de conocimiento en la Historia parece haber seguido un derrotero muy diferente: ya lo decía Kant al quejarse de que en la Metafísica, al parecer cada autor pretende comenzar nuevamente de cero (Cf. 19). Por otra parte, la sumatoria de estos modestos papers, de un grupo de humildes hipótesis escasamente desarrolladas, no constituye una gran tesis con pretensiones mayores, ni siquiera cuando el autor es el mismo. En efecto, se pueden sumar diferentes artículos de autores diversos o del mismo autor sobre algún tema o problema específico, sin embargo, el resultado no es propiamente un trabajo de repercusiones y profundidad mayores. Una suma de partes no constituye necesariamente un todo: puede quedar simplemente en una suma de partes. En tercer lugar, la "claridad", en un sentido bastante cartesiano, es una característica propia de un paper. "A good Philosophy paper is modest and makes a small point; but it makes that point clearly (...)" (Pryor) se lee en un "manual" acerca de cómo escribir un paper filosófico. En un paper, insisten los textos de este tipo, solo se debe decir "exactamente" lo que se pretende expresar, minimizando al máximo cualquier posibilidad de malos entendidos. Quienes confeccionan estos instructivos para escribir papers no dejan de recalcar hasta el cansancio que la claridad y la precisión son elementos esenciales: si un texto no cuenta con estas características simplemente no es

11 "En este trabajo he puesto la mayor atención en la exhaustividad y me atrevo a decir que no hay un solo problema metafísico que no haya quedado resuelto o del que no se haya ofrecido al menos la clave para resolverlo" (Kant 10). 
un buen paper ${ }^{12}$. Vinculado con esta exigencia, el paper debe ser "conciso" e ir "directamente al grano". Las introducciones que busquen poner al lector no informado en antecedentes sobre el tema tratado, las justificaciones acerca de la importancia del tema, las contextualizaciones que expliciten el marco de problematización tanto histórico como teórico, en general todo tipo de "digresión" debe ser evitada. Algunos autores incluso recomiendan eliminar las introducciones definitivamente ${ }^{13}$. Cualquier explicación o disquisición que se desvíe del punto central del paper, aunque aporte a la comprensión del tema, debe ser evitada por superflua. El lema sería algo así como: "Get right down to business! Avoid inflated, rhetorical introductory remarks (commonly known as "fluff") ") (Portmore).

"En general -señala Cecilia Sánchez-a quien escribe en este formato se le exige ser informativo y evitar digresiones, detenciones, desvíos y detalles, cuestión que para la Filosofía es en extremo nociva. Piénsese en Platón, Nietzsche, Derridá, Helene Cixous o Luce Igigaray; en el caso nacional se puede nombrar a Humberto Giannini, Patricio Marchant, Pablo Oyarzún y Olga Grau. En todos estos casos, como en otros, la digresión es parte del pensamiento" (383). Esta acertada observación lleva a sostener que el "irse por las ramas" ha sido y es, en el ámbito de la Filosofía en particular, pero también en el de la Humanidades en general, parte del proceso de pensamiento, un aspecto de su desarrollo, por lo que la limitación impuesta por el estilo paper estaría en contradicción con ello.

Lo que se busca con la claridad, con la eliminación de las digresiones, con el ser lo más conciso y directo posible, incluso con la exigencia de ser modesto en las pretensiones es, en definitiva, la "eficiencia", lo que constituye, sin duda, otro de los rasgos más característicos del paper. Esto, como señala Delgado, se relaciona con la manera en que se entiende hoy el conocimiento científico, en donde "la velocidad del tráfico de ideas" es un elemento central ${ }^{14}$. Todo lo relacionado con el mundo del paper es "rápido":

12 "You must say exactly what you mean and in a way that minimizes the chances of being misunderstood. It is difficult to overemphasize this point. There is no such thing as a piece of good philosophical writing that is unclear, ungrammatical, or unintelligible. Clarity and precision are essential elements here" (Horban).

13 "Introductions should be as brief as possible. In fact, I recommend that you think of your paper as not having an introduction at all. Go directly to your topic" (Ídem).

14 "Los nuevos conocimientos científicos son realizados por muchas más personas que antes y en menos tiempo. Por consiguiente, se necesitaba un medio de comunicación que diese mayor velocidad al tráfico de ideas" (Delgado y Ruiz Pérez). 
una idea prontamente se transforma en un escrito, pues su factura no presenta mayores dificultades, un escrito es enviado de inmediato a una revista que tiene plazos perentorios para evaluar y compromisos de publicación anual, y en pocos meses el paper comienza a circular. Para comprender esta vertiginosa circulación de papers basta con ponerla en relación con los años que puede tomar la confección de un libro: el estudio conducente a las ideas que sirven de marco, su escritura, la redacción de los capítulos, la edición y corrección del manuscrito, la publicación misma y la puesta en circulación. La introducción de paper en el ámbito de la Filosofía y de las Humanidades ha significado una aceleración brutal.

\section{CONFLICTO}

Aclarado en general qué es lo que se dice cuando se dice "paper", retomo lo que venía sosteniendo: el paper es un caso paradigmático de esa "logofobia" de la que hablaba Foucault en cuanto a que constituiría un claro intento por controlar la peligrosidad del discurso humanista, de dominar su proliferación, de organizar su incontrolabilidad. La hipótesis que sostengo es que la instalación concertada del paper como género literario preponderante obedece, justamente, a la necesidad de controlar la producción del discurso en Humanidades. Dicho de otra forma, sostengo que el paper mismo y su instalación como formato prioritario es uno de aquellos procedimientos destinados a conjugar, dominar y esquivar las peligrosidades propias del discurso humanista. La manera en que se lleva a cabo es mediante una serie de prohibiciones, barreras, límites y reglas que encauzan, guían y limitan la producción discursiva. Con ello se lograría controlar el temor al discurso, pues se lo mantendría a raya, dentro del campo delimitado del paper. En lo relativo a dichas reglas, lo inmediatamente destacable es el cúmulo de prohibiciones que acompaña a este género y que, en la práctica, tiene como consecuencia inmediata dejar fuera del ámbito del discurso lo más interesante y sustancial de la producción intelectual latinoamericana, abandonándola, sin más, al terreno de lo monstruoso, del ruido que no es escuchado. Un par de observaciones acerca de estas prohibiciones podrían servir para ir vislumbrando las consecuencias que está teniendo sobre nuestra labor esta tiranía del paper.

En primer lugar, es relativamente evidente que el paper establece una serie de prohibiciones respecto del sujeto, es decir, límites y barreras en lo referente a quien sea su autor. De lo que se trata aquí es fundamentalmente 
del hecho de que no cualquiera puede escribir un paper. El mismo formato limita las posibilidades de su autoría. Quisiera referirme, para comenzar, al dispositivo de exclusión de cierta palabra, de la palabra de "algunos", por ser considerada "nula y sin valor", pues "no contiene ni verdad ni importancia" (Foucault, Orden 16), es decir, discursos que no son "reconocidos ni escuchados" y que "regresa[n] al ruido". La lista de los sujetos excluidos del discurso de las Humanidades en general y del paper en particular es larga. No se trata tan solo de la palabra del loco - como diría Foucault-a la que se le ha despojado de su valor de verdad. En las mismas condiciones están la palabra del amateur, del autodidacta, pero también durante siglos, milenos, la de la mujer y, sin duda también, la de los pertenecientes a las culturas no europeas: africanos, indios, chinos, etc.

Paradigmático es el caso del discurso filosófico de los latinoamericanos, cuyo valor de verdad ha sido tan sistemáticamente negado que, por lo mismo, se le ha considerado simplemente como un "ruido": una secuencia de sonidos ininteligible, carente de armonía, sin sentido alguno, por lo tanto, desechable. Este punto ha sido ya largamente puesto de manifiesto por los autores de la segunda mitad del siglo XX, por lo que no ahondaré en ello. Quisiera solo detenerme ahora, muy brevemente, en una observación relativa a lo que ocurre hoy con nuestro idioma en el contexto de la invasión del paper. Digamos, para comenzar provocando, que el español simplemente no es una lengua de paper. Como tal vez algunos saben, la expresión alemana para decir que simplemente no se entiende nada es "suena a español", tal como nosotros decimos que "parece chino". Es el inglés, a todas luces, la lengua del paper. Las universidades, al menos en Chile, ofrecen, de hecho, un servicio gratuito de traducción de nuestros papers al inglés: lengua a la que se vehicula la verdad científica. Aunque se nos acepte un texto en español, sin embargo, siempre debe ser precedido por un abstract (en inglés). En este punto no deja de ser sintomático, por ejemplo, que de las 141 revistas de Filosofía incluidas en el catálogo ISI, solo 8 se publican en países hispanohablantes y de ellas, 6 en España ${ }^{15}$. En el contexto mundial de la ciencia-paper, lo escrito en español sigue siendo, en gran medida, ruido, es decir, un discurso que no es reconocido ni escuchado. 
Ahora bien, aunque no fuéramos segregados a priori por nuestra calidad de locos, enfermos, por razones de género o de creencias, por el lugar de nacimiento, la cultura de procedencia o la lengua en que hablamos, aun así no todos pueden escribir un paper, pues para ello se requiere de cierto entrenamiento, un proceso de formación que cualifique al individuo para hacerlo. Escribir este tipo de textos, por lo tanto, es solo para aquellos que conocen las reglas para confeccionarlo y, en consecuencia, tienen la habilidad para hacerlo ${ }^{16}$. En términos foucaultianos se trata de poder entrar al orden del discurso filosófico, donde solo algunos sujetos pueden hablar. Quienes pueden hacerlo son aquellos que han sido debidamente entrenados y consecuentemente acreditados. La acreditación es algo que otorgan los que ya están habilitados. Se trata de lo que se ha denominado la "autonomía" en el ejercicio de la profesión: los colegas realizan un ejercicio monopólico sobre el desarrollo de la actividad. Ellos son los que enseñan, forman, acreditan y luego evalúan.

Tanto los comités editoriales de las revistas como los evaluadores o réferis externos velan porque el discurso presentado dé cuenta, entre otras cosas, de que el autor tiene la calificación necesaria, es decir, el suficiente dominio del orden discursivo como para que sea posible la publicación. De lo contrario, simplemente se rechaza. Basta con asomarse a mirar los formularios que las revistas mandan a sus evaluadores, donde habitualmente se encuentran consultas acerca de, por ejemplo, el manejo de bibliografía, el cumplimiento de las formalidades, el uso del lenguaje, el orden y claridad del texto, etc. Se avalúa, en el fondo, el cumplimiento de lo que Foucault llama el "ritual", es decir, un conjunto de signos que deben acompañar al discurso y de cuya satisfacción depende que dicho discurso sea o no escuchado. El paper está plagado de rituales y solo quienes los conozcan, los que demuestren que los manejan, tienen posibilidad de publicar.

Los ensayistas, los “originales", los autodidactas, aquellos sujetos que, sin tener una formación sistemática ni contar con la acreditación correspondiente, -y que tienen un lugar central en la tradición de las Humanidades en América Latina, aportando con la potencia de su reflexión- quedan así del todo marginados, su voz es simplemente desautorizada. Para captar el tremendo límite que esto impone hay que tener en cuenta, aquí, que los estudios

16 (...) se trata de determinar las condiciones de su utilización, de imponer a los individuos que los dicen cierto número de reglas y no permitir de esta forma el acceso a ellos a todo el mundo" (Foucault, Orden 38-39). 
sistemáticos, universitarios, normalizados en Humanidades datan, en el continente, desde la primera mitad del siglo XX y tienen, por lo tanto, menos de un siglo de existencia. Si a esto se agrega el hecho de que una enseñanza sistemática en lo referente a la producción de papers aún no se da en la gran mayoría de nuestras instituciones, la conclusión es que los latinoamericanos quedan prácticamente fuera del circuito de los papers: solo la voz de los más jóvenes, los recientemente formados, los formados en otras latitudes tiene hoy posibilidad de ser escuchada.

En el marco de las ritualidades de las que venimos hablando es claro que el paper instala una serie de exigencias, de reglas muy estrictas respecto de su forma, lo que en la práctica se traduce en que no se puede decir lo que se pretende decir de cualquier manera. Existe un modo-paper de escribir y dicho modo particular de escritura está rigurosamente normalizado. En las "normas de publicación" que las revistas publican y publicitan se consignan, en primer lugar, asuntos formales: idioma en que debe estar el escrito para ser aceptado, extensión máxima (e incluso mínima) de los textos, formas de citación (APA o MLA), formas de numeración, formato del título, resumen (abstract), incorporación de palabras clave (normalmente 5), bibliografía de acuerdo con un formato establecido, interlineado, incluso tipo de letra y tamaño de la misma. También el modo de escritura está normado. Reglas al respecto son, por ejemplo, la utilización de ciertas estrategias discursivas y la sanción de otras. Se puede mencionar aquí, en primer lugar, el exilio del uso de la primera persona del singular. En este mismo sentido, se puede aludir, en segundo lugar, a que debe quedar fuera cualquier tipo de opinión infundada, así como toda impresión, manifestación emotiva o afectiva. En tercer lugar, se puede comentar la necesaria marginación de todo relato anecdótico, coyuntural, vivencial.

Otras exigencias que se imponen al discurso del paper tienen relación con el uso de un lenguaje académico o formal, que evita y excluye cualquier resto de lenguaje cotidiano o vulgar. La ironía, la broma, los giros propios de un lenguaje suelto o coloquial, la alusión o el simple comentario al margen quedan excluidos. Se trata de la exigencia de un lenguaje parco, seco y con pretensiones - por eso mismo- de seriedad, claridad y falta de ambigüedad. Se exige un uso técnico de la palabra que tiene como consecuencia, entre otras, que solo podrá ser comprendido por los iniciados en ese modo de lenguaje. Todo tipo de retórica o poética quedan marginadas. No hay espacio para los lujos estilísticos, los alardes de buen escritor. Debe evitarse, dentro de lo posible, el uso de cualquier tipo de metáforas, de símiles, de figuras. 
El discurso de los intelectuales y pensadores latinoamericanos, en lo que tiene de más original, innovador, propositivo, simplemente no cabe en el marco de todas estas restricciones formales. Un discurso en el que el sujeto siempre está presente -que si seguimos a Roig, tiene que hacerse presente-, un discurso para el cual el contexto, la circunstancia, la situación históricocultural es parte de su contenido y de su sentido como lo es en el caso de una Filosofía entendida como "auroral"; un discurso en el que lo literario, el mostrar estético y ameno interrumpe e incluso suplanta la argumentación estricta y árida. Un discurso con estas características es sistemática e invariablemente rechazado por las revistas que publican papers. Nuestra América de José Martí nunca habría sido bien evaluada por los réferis, así como tampoco lo sería Calibán de Fernández Retamar o El Laberinto de la Soledad de Octavio Paz. Al Facundo de Sarmiento lo habrían rechazado, sin lugar a dudas, por su extensión y por su falta de argumentación y lo mismo ocurriría con el Ariel de Rodo y el Evangelio Americano de Francisco Bilbao, entre tantos otros clásicos del pensamiento latinoamericano. El punto es que estos discursos simplemente no caben en el estricto y reglado formato del paper, pues, como bien ha hecho ver Christiane Schildknecht, hay una "(...) conexión sistemática entre cada concepto del conocimiento o del saber filosófico y la forma (literaria) en que se expresa o se transmite dicho saber" (21). En otras palabras, no se puede decir todo de cualquier manera. En términos prácticos, el control sobre la forma de decir implica directamente el que se ejerza también sobre el contenido; y el discurso del pensamiento humanista latinoamericano, en su vertiente más interesante, es un discurso que no se deja controlar y, por ello, hoy paga con la segregación y la sordera.

Un paper contiene, en tercer término, rigurosas exigencias respecto de su estructura: siempre debe haber una "hipótesis" y una "demostración", y dicha demostración debe ser eminentemente de tipo argumentativo. Esto se refleja en las "normas de publicación" de las revistas cuando se impone expresamente que el discurso esté organizado en tres momentos: introducción, desarrollo y conclusiones. El discurso del paper no puede ser entonces más que "proposicional": es un discurso del "decir" alejado de todo rastro de retórica, de Literatura. Cualquier otra estructura, otro tipo de discurso noproposicional, por lo tanto, está prohibido, lo cual no significa, sin duda, que no existan otras estructuras posibles para un discurso. Christiane Schildnecht hace ver que históricamente se abrieron dos caminos del conocimiento para el pensamiento filosófico, ligados a dos modos de exposición: 1. "El camino trazado por la ciencia, directo, argumentativo (...)" y 2 . "El camino indirecto, 
mostrativo -indicado por la Literatura-, basado en sentido estricto en formas literarias de exposición" (23). La autora hacer ver, sin embargo, que desde que Platón exilió a los poetas de la Polis se ha impuesto, hasta hoy, el primer modo. Esta observación, que se extiende sin dificultad a todo el ámbito de las Ciencias Humanas o del Espíritu, se termina de sancionar con instalación de paper. En un paper no hay lugar para el simple "mostrar", sino solo para el definitivo "de-mostrar".

Lo que tenemos en nuestra tradición de pensamiento latinoamericano, sin embargo, son fundamentalmente discursos que se instalan en modos no argumentativos ni proposicionales de exposición, acercándose fuertemente al modo literario de conocimiento en el que el saber no se encuentra expresado directamente en los textos, sino que se trasmite a través de ellos; es mostrado, no dicho. En nuestros clásicos no es tanto la argumentación lo que prima, sino más bien la "puesta en escena" en busca de persuasión. De allí la utilización de metáforas, figuras, anécdotas, situaciones puntuales, relatos, etc., junto a un lenguaje, a una retórica que además de estética, busca cautivar al lector, seducirlo, persuadirlo. Una vez más se podrían mencionar aquí los discursos de un Martí, Sarmiento, Bilbao, Paz, etc.

Otro orden de prohibiciones impuestas por el paper atañen su objeto. En un paper no se puede hablar de todo: hay, en primer lugar, asuntos, temas que no se tocan, que no pueden ser abordados, pero también hay partes o escorzos de temas o asuntos aceptados que no pueden ser mencionados. Quienes nos dedicamos profesionalmente hoy a alguna de las áreas de las Humanidades sabemos bien que esto es así, por lo que, en general, tenemos la precaución de no escribir papers sobre temas vetados, que van a ser evidentemente rechazados. Una vez más, sin embargo, ante la pregunta por cuáles son dichos temas, cuáles son concretamente las prohibiciones, la respuesta tiende a ser el silencio. Sabemos que existen, conocemos sus alcances, pero no sabemos señalarlos. Todo funciona aquí como si la norma, la prohibición fuera tácita. No está escrita en ninguna parte, pero sin duda actúa y, en general, vislumbramos cómo lo hace.

Foucault propone una distinción entre "discursos fundamentales y creadores", por una parte, y "discursos que solo repiten, glosan o comentan", por la otra. Quisiera agregar a esta diferencia los "discursos críticos, destructivos y denunciantes". El paper, en tanto se caracteriza por ser efímero, modesto y eficiente, no tiene la pretensión de constituirse en un discurso fundamental, menos aún fundacional, y solo en marcadas ocasiones podría aspirar a ser moderadamente creador. En este punto resulta iluminadora la investigación 
de Ganz y Shepherd, quienes analizaron el caso de los premios Nobel en Economía. De acuerdo con sus resultados, de los 20 ganadores vivos en ese momento, solo a tres nunca se les había rechazado un artículo, pero lo más sorprendente es que entre los otros 17 fue habitual la convicción de que los textos que les fueron rechazados eran justamente los que ellos consideraban sus mejores trabajos y figuraban entre sus obras más citadas, es decir, lo que se les rechazó fueron fundamentalmente aquellos discursos que tuvieron finalmente un sentido fundacional y creador. En el mismo sentido, Campanario hace una lista de 36 casos de autores cuyos papers fueron rechazados por diferentes revistas en distintas disciplinas y que luego fueron recompensados con el premio, justamente en virtud de esos trabajos.

Así como no es fundacional, el del paper no es tampoco un discurso crítico, destructivo, ni busca desestabilizar nada: su objetivo expreso y su límite es aportar humildemente un grano de arena al avance del conocimiento ya instalado. En este contexto es que su aspiración es ser un buen "comentario" o una "glosa" inteligente que cumpla con las exigencias del formato. Se trata de un texto acerca de textos: el paper tiene como objeto de trabajo otros discursos a los que comenta, a los que glosa, en una palabra, a los que repite". Esto se da, sin duda, de diferentes formas: hay papers acerca de una obra de un autor, otros sobre un asunto que atraviesa varias obras de diferentes autores, etc. El paper no pasa de ser un meta-discurso.

Nuevamente los latinoamericanos y sus discursos rompen con esta exigencia, en tanto que la pretensión fundacional y crítica está siempre allí, siempre presente; es lo que lo anima, lo que le da realidad. Ya lo decía Arturo Roig: "(...) el pensamiento latinoamericano, en lo que muestra de verdaderamente creador, se nos aparece cuestionando el discurso colonialista” (Roig 79). La historia completa del pensamiento latinoamericano podría ser leída en esta clave: como lucha por la emancipación. Para este discurso no hay lugar en el ámbito del paper. Desde Las Casas, pasando por Bilbao, Lastarria, Sarmiento, Mariátegui, Martí, Rodó, hasta Salazar Bondy, Zea, Kusch, Dussel, Roig, Millas, Cordua, Rivano: todos críticos, todos denunciantes, todos emancipadores.

\section{DESENLACE}

Sostenía Foucault muy acertadamente que hay un vínculo evidente entre la existencia histórica de una cierta "voluntad de saber, de verdad" y la forma 
que ese saber tiene de ponerse en práctica en la sociedad, cómo es valorado, distribuido, repartido (Orden 22). Hoy, como se ha visto, el paper es la forma en que una cierta "voluntad de verdad" se instala institucionalmente en la sociedad y ejerce, como dice el francés, “(...) sobre los otros discursos (...) una especie de presión y de poder de coacción" (Orden 22). En este momento asistimos, sin duda, a dicha presión, una que nos impele a concentrar nuestro trabajo en la producción de papers. Lo central, sin embargo, es vislumbrar las consecuencias que esto tiene para el trabajo intelectual en general y las repercusiones que representa para nuestra tradición de pensamiento. Es indispensable tomar conciencia de las presiones, de los límites que se nos están imponiendo, pues dichas barreras no son ni casuales, ni anecdóticas; menos aún azarosas o poco importantes. Se trata de la sistemática imposición de una estructura que permita controlar el discurso, que lo mantenga dentro de márgenes que acallen su voz más sorprendente y que silencie lo más rupturista o fundacional.

\section{BIBLIOGRAFÍA}

Bonilla, Alcira B. "La biografía como género filosófico: construcción de subjetividad, memoria y responsabilidad”. Strómata LXIV/ 12 (2008): 39-52.

Campanario, Juan Miguel. "El sistema de Revisión por expertos (peer review); muchos problemas y pocas soluciones". Revista Española de Documentación Científica. 25/3 (2002): 166-184.

"Consolation for the scientist: Sometimes it is hard to publish papers that are later highly-cited”. Social Studies of Science. 23 (1993): 342-62.

Delgado López-Cózar, Emilio y Ruiz Pérez, Rafael. "La comunicación y edición científica: fundamentos conceptuales". Homenaje a Isabel de Torres Ramirez: Estudios de documentación dedicados a su memoria. Granada: Editorial Universidad de Granada, 2009. 131-150.

Foucault, Michel. El orden del discurso. Alberto González T. (trad.). Barcelona: Ediciones Fábula, 2002.

La Arqueología del Saber. 1970. México: Siglo Veintiuno, 2001.

Gabriel,Gottfried; Schildknecht, Christiane (edit.). Literarische Formen der Philosophie. Stuttgart: J.B. Metzlersche Verlagsbuchhandlung, 1990.

Ganz, J.S. y Shepherd. G.B. "How are the mighty fallen; Rejected classic articles by leading economist". Journal of Economic Perspectives 8 (1994): 165-79.

Kant, Inmanuel. Critica de la razón pura. Pedro Ribas (trad.). España: Ediciones Alfaguara. 1993. Le Goff, Jacques. Pensar la Historia. 1977. Marta Vasallo (trad.). España: Altaya, 1995. 
Mainer, J. C. "Géneros literarios y géneros filosóficos: una frontera permeable". Literatura y Filosofía en la crisis de los géneros. Cuadernos de la Fundación Juan March 4 (1999): 19- 48.

Marías, Julián. "Los géneros literarios en filosofía", Obras. IV. 1953. Madrid: Revista de Occidente, 1969. 331-354

Ossandón B., Carlos. "Investigación en Humanidades. Escrituras y Protocolos". Debates Críticos 2 (2011):36-38.

Pizarro Cortés, Carolina. “¿Debería aceptar yo sin más, las paparruchas y embustes de vuestros cronistas? Alpha 30 (2010): 215-230.

"El descentramiento del sujeto en el imaginario independentista latinoamericano". Journal of Latin American Cultural Studies 20/4 (2011): 323-324.

Nuevos Cronistas de Indias. Sevilla: Thémata, 2012.

Roig, Arturo Andrés. Caminos de la Filosofía Latinoamericana. Venezuela: Universidad de Zulia, 2001.

Ricoeur, Paul. Tiempo y narración. México: Siglo XXI, 1995.

Historia y narratividad. España: Paidós, 1999.

Santos Herceg, José. "De espejismos y fuegos fatuos. Publicar filosofía hoy en Chile (ISI y Scielo)”. La Cañada. Revista del pensamiento filosófico chileno. 1 (2010): 126-147.

"Filosofía de Mercado. El filósofos profesional como MINI-PYME". Paralaje 7 (2012): 45-63.

Sánchez, Cecilia. "Institucionalidad de la filosofía: entre la reflexión y el conocimiento productivo". Mapocho 67 (2010): 373-385.

Schildknecht, Ch. "Entre la ciencia y la literatura: formas literarias de la filosofía". Figuras del Logos. López de la Vieja (ed.) México: FCE, 1994. 21-40.

Spinak, Ernesto. "Indicadores cienciométricos". ACIMED 9 (Supl. 1) (2001):42-49

White, Hayden. Metahistory: The Historical Imagination in Nineteenth-Century Europe. Baltimore: The Johns Hopkins University Press, 1973. (Metahistoria. México: FCE, 1992).

The Content of the Form: Narrative Discourse and Historical Representation, Baltimore: The Johns Hopkins University Press, 1987. (El contenido de la forma. España: Paidós, 1992)

Zachery Seech. Writing Philosophy Papers. Belmont: CA: Wadsworth, 1997

Manuales, Instructivos y otros

Berkeley, Istvan, "How to Write a Philosophy Paper"

http://www.cariboo.bc.ca/ae/php/phil/mclaughl/courses/howrit.htm

Cameron, Rich, "The Structure of a Typical Philosophy Paper"

http://www.colorado.edu/philosophy/fac/cameron/ethics1100/ho.rfww.html. 
Harold, James "On Writing Philosophy Papers"

http://www.tc.umn.edu/ harol006/paper.html.

Portmore, Douglas W., "Tips on writing a Philosophy paper"

http://www.public.asu.edu/ dportmor/tips.pdf

Pryor, James "Guidelines on Writing a Philosophy Paper"

http://www.princeton.edu/ jimpryor/general/writing.html

http://www.jimpryor.net/teaching/guidelines/writing.html

Watson, Ellen, “A Guide for Writing Papers in Philosophy”

http://www.uq.oz.au/ pdgdunn/watessay.html

"Writing A Philosophy Paper"

http://www.sfu.ca/philosophy/writing.html 